\title{
Cefditoren in upper and lower community-acquired respiratory tract infections
}

\author{
This article was published in the following Dove Press journal: \\ Drug Design, Development and Therapy \\ 9 February 2011 \\ Number of times this article has been viewed
}

\author{
Francisco Soriano' \\ María-José Giménez ${ }^{1,2}$ \\ Lorenzo Aguilar ${ }^{1,2}$ \\ 'PRISM-AG, Madrid, Spain; \\ ${ }^{2}$ Microbiology Department, School \\ of Medicine, University Complutense, \\ Madrid, Spain
}

\begin{abstract}
This article reviews and updates published data on cefditoren in the evolving scenario of resistance among the most prevalent isolates from respiratory tract infections in the community (Streptococcus pyogenes, Haemophilus influenzae, and Streptococcus pneumoniae). By relating the in vitro activity of cefditoren (in national and multinational surveillance and against isolates with emerging resistant genotypes/phenotypes) to its pharmacokinetics, the cefditoren pharmacodynamic activity predicting efficacy (in humans, animal models, and in vitro simulations) is analyzed prior to reviewing clinical studies (tonsillopharyngitis, sinusitis, acute exacerbations of chronic bronchitis, and community-acquired pneumonia) and the relationship between bacterial eradication and clinical efficacy. The high in vitro activity of cefditoren against the most prevalent respiratory isolates in the community, together with its pharmacokinetics (enabling a twice daily regimen) leading to adequate pharmacodynamic indexes covering all $S$. pyogenes, H. influenzae, and at least $95 \%$ S. pneumoniae isolates, makes cefditoren an antibiotic that will play a significant role in the treatment of respiratory tract infections in the community. In the clinical setting, studies carried out with cefditoren showed that treatments with the $400 \mathrm{mg}$ twice daily regimen were associated with high rates of bacteriological response, even against penicillin-nonsusceptible $S$. pneumoniae, with good correlation between bacteriological efficacy/ response and clinical outcome.
\end{abstract}

Keywords: cefditoren, Streptococcus pyogenes, Haemophilus influenzae, Streptococcus pneumoniae, community-acquired respiratory tract infections

\section{Scenario for new antibiotics for the treatment of respiratory tract infections}

Infections, mainly respiratory tract infections, ${ }^{1}$ are the most frequent reason for seeking medical attendance in the community. ${ }^{2}$ Around $85 \%$ to $90 \%$ of global antibiotic consumption occurs in the community, with $80 \%$ of this consumption for the treatment of respiratory tract infections. ${ }^{3}$ Streptococcus pyogenes causing pharyngotonsillitis, and Streptococcus pneumoniae and Haemophilus influenzae causing otitis and lower respiratory tract infections (acute exacerbations of chronic bronchitis and communityacquired pneumonia), are the most prevalent isolates in community-acquired respiratory tract infections, and can be considered index bacteria for assessing antibiotic resistance.

Several studies carried out in Spain have shown that antibiotic consumption is the main reason for resistance selection in the community. ${ }^{4}$ These studies related consumption of $\beta$-lactams (mainly second-generation oral cephalosporins) and macrolides (mainly long half-life macrolides) with penicillin/erythromycin resistance in S. pneumoniae
Correspondence: Francisco Soriano

Avda. Osa Mayor 136,

28023 Madrid, Spain.

Tel +34 630619376

Email fsoriano9@yahoo.com 
(with temporal ${ }^{5}$ and geographical ${ }^{6}$ associations) and with ampicillin/amoxicillin resistance in $H$. influenzae (this study showed higher accountability for aminopenicillins with or without clavulanic acid). ${ }^{7}$ Other studies showed that macrolide consumption (mainly long half-life macrolides) was linked to erythromycin resistance in $S$. pyogenes. ${ }^{8,9}$

The Spanish data show that the analysis of resistance in the community should be seen as a global problem, since geographical and temporal relationships between resistance in these target bacteria have been described, leading to the concepts of co-resistance selection and co-selection of resistance. Due to co-resistance selection, there is a significant association between penicillin and erythromycin resistance in S. pneumoniae, ${ }^{10,11}$ and because of the co-selection of resistance, the penicillin/erythromycin resistance in $S$. pneumoniae is associated with ampicillin resistance in $H$. influenzae. ${ }^{10}$ Further, this resistance in $S$. pneumoniae and in $H$. influenzae is significantly related to erythromycin resistance in $S$. pyogenes from a geographical perspective. ${ }^{10}$ The geographical relationship between macrolide resistance in S. pneumoniae and in S. pyogenes in Spain emphasizes the idea of resistance as a global problem, since $S$. pneumoniae is isolated mainly from adults, from lower respiratory tract samples, with $\mathrm{MLS}_{\mathrm{B}}$ as the prevalent resistance phenotype, while $S$. pyogenes is isolated mainly from children, from pharyngotonsillitis, with M-efflux as the prevalent resistance phenotype. ${ }^{10,11}$

The need for new antibiotics is defined mainly by their antimicrobial activity against the prevalent resistance phenotypes, rather than the activity against phenotypes susceptible to antibiotics previously used in the community. The analysis of resistance prevalence and phenotypes reported in index bacteria will define the context in which new antibiotics should demonstrate in vitro activity.

\section{S. pyogenes}

S. pyogenes is highly susceptible to penicillin (strains with minimum inhibitory concentration [MIC] $>0.12 \mu \mathrm{g} / \mathrm{mL}$ have not been found) and should be considered susceptible to all $\beta$-lactams. In contrast, resistance to erythromycin is widely reported, with geographical variations in prevalence rates and predominant resistance phenotypes. The rate of erythromycin resistance in $S$. pyogenes varies among countries, from $6.9 \%$ in the US (56\% $\mathrm{MLS}_{\mathrm{B}}$ phenotype) $)^{12}$ to $25.6 \%$ in Hong Kong. ${ }^{13}$ In a recent study performed in Eastern Europe, the rate of erythromycin resistance in S. pyogenes was low $(<10 \%)$ in Romania and Baltic countries, intermediate (10\%-20\%) in Poland and the Czech Republic, and high $(>25 \%)$ in Hungary and Slovakia. ${ }^{14}$ The predominant $(75 \%)$ resistance phenotype was $\operatorname{MLS}_{\mathrm{B}}(58.9 \%$ of the constitutive phenotype plus $16.1 \%$ of the inducible phenotype). ${ }^{14}$ In Spain, although the rates of erythromycin resistance have decreased in SAUCE (Susceptibility to the Antimicrobials Used in the Community in España) studies over recent years (26.7\% in 1996-1997, $20.4 \%$ in $1998-1999,24.3 \%$ in $2001-2002$, and $19.0 \%$ in 2006-2007), no temporal decreasing trend for erythromycin resistance was found in contrast to an increasing trend in the percentage of the $\mathrm{MLS}_{\mathrm{B}}$ phenotype $(7.0 \%, 10.5 \%, 14.0 \%$, and $35.5 \%$, respectively, among resistant strains in the corresponding SAUCE studies). ${ }^{15}$

Since both mechanisms of resistance (M-efflux and $\mathrm{MLS}_{\mathrm{B}}$ ) imply resistance to 14- and 15-membered macrolides, erythromycin resistance implies resistance to azithromycin and clarithromycin. ${ }^{16}$

\section{$H$. influenzae}

Resistance to $\beta$-lactams in $H$. influenzae is defined using ampicillin as a marker of resistance. Most ampicillin-resistant isolates are $\beta$-lactamase producers: TEM-1, TEM-2 and, with lower frequency, ROB-1. The remaining ampicillin-resistant isolates are resistant due to mutations in the $f t s I$ gene causing alterations in the amino acid sequences of penicillin-binding protein 3 (PBP3). Resistance phenotypes showing mutations in the $f$ tsI gene are BLNAR ( $\beta$-lactamase negative ampicillin resistant) and BLPACR ( $\beta$-lactamase positive amoxicillin/ clavulanic acid resistant), which exhibits both resistance mechanisms ( $\beta$-lactamase production and mutations in the ftsI gene). These phenotypes should be considered also resistant to amoxicillin/clavulanic acid, ampicillin/sulbactam, piperacillin/tazobactam, cefaclor, and cefuroxime. ${ }^{17}$

The epidemiology of ampicillin resistance varies geographically and temporally. In a global surveillance including 15 countries (in 2003-2004), ampicillin resistance ranged from $8.7 \%$ in South Africa to $29.6 \%$ in Asia. ${ }^{18}$ In the US, ampicillin resistance averaged about $30 \%$ in the period 2001-2005. ${ }^{19}$ In a European surveillance (2004-2005) including isolates from 11 countries, mean ampicillin resistance was $16.4 \%$, resistance due to $\beta$-lactamase production ranging from $17.6 \%$ in France to $0 \%$ in Germany and Netherlands, and BLNAR isolates ranging from 33.9\% in Spain to $0 \%$ in France and Netherlands. ${ }^{20}$ In Spain (SAUCE studies: 1996-1997, 1998-1999, 2001-2002, 2006-2007) ampicillin resistance seems to be decreasing over time $(38.6 \%, 30.0 \%, 25.1 \%$, and $16.1 \%$, respectively), both resistance due to $\beta$-lactamase production $(25.7 \%, 23.0 \%, 20.0 \%$, and $15.7 \%$, respectively) and to the BLNAR phenotype 
$\left(13.5 \%, 12.0 \%, 4.4 \%\right.$, and $0.7 \%$, respectively).${ }^{15}$ However the rate of BLNAR and BLPACR among strains exhibiting high MIC of ampicillin ( $\geq 1 \mu \mathrm{g} / \mathrm{mL}$ ) or amoxicillin/clavulanic acid $(\geq 2 / 1 \mu \mathrm{g} / \mathrm{mL})$ is higher, as shown in a Spanish multicenter study carried out in 2005-2007 where among the 196 strains with high MICs identified, 165 showed mutations in the ftsI gene (73\% BLNAR and 27\% BLPACR). ${ }^{21}$ The situation in Japan is the most worrisome since in nationwide global surveillances the percentage of BLNAR and BLPACR strains was $29.1 \%$ and $6.7 \%$, respectively, in adults in $2007^{22}$ and $59.3 \%$ and $6.4 \%$, respectively, in children in $2004 .^{23}$

According to the SAUCE study in 2006-2007, similar susceptibility rates to ampicillin or amoxicillin/clavulanic acid were obtained by applying CLSI (Clinical and Laboratory Standards Institute) and PK/PD breakpoints (pharmacodynamic [PD] breakpoints predicting eradication that are obtained through the relationship between pharmacokinetic [PK] parameters and MIC).$^{15}$ In contrast, rates of susceptibility to cefaclor changed from $97.8 \%$ (by applying CLSI breakpoints) to $25.8 \%$ (PK/PD breakpoints). ${ }^{15}$ Similarly, percentages of susceptibility to macrolides were high by applying CLSI breakpoints (99.3\% and 100\% to clarithromycin and azithromycin, respectively) but sharply decreased to $5.1 \%$ and $23.8 \%$, respectively, when PK/PD breakpoints were considered, ${ }^{15}$ probably due to the presence of efflux pumps in virtually all $H$. influenzae strains. This suggests that current CLSI breakpoints for $H$. influenzae for macrolides should be reviewed. ${ }^{24}$

\section{S. pneumoniae}

Except for fluoroquinolones (worldwide nonsusceptibility rates to levofloxacin are as low as $<2.5 \%{ }^{25}$ ), antibiotic resistance in $S$. pneumoniae also depends on geographic location and time, antibiotic pressure (consumption) and the introduction of the 7-valent conjugate vaccine (PCV7 including serotypes most associated with penicillin/erythromycin nonsusceptibility) being the principal influencing factors. In a worldwide surveillance study, penicillin nonsusceptibility was $66 \%$ in South Africa, $47.3 \%$ in Asia-Pacific, $44.1 \%$ in the Middle East, $42.1 \%$ in North America, 37.9\% in Latin America, and $27.9 \%$ in Europe. ${ }^{26}$ Penicillin (with CLSIdefined breakpoints for oral penicillin) is the best epidemiological marker since nonsusceptibility rates to $\beta$-lactams and to macrolides are clustered in penicillin-intermediate and/or in penicillin-resistant $S$. pneumoniae ${ }^{27}$ In this sense, in a 2001-2002 surveillance study (mainly including noninvasive isolates) in Spain the reported nonsusceptibility rates to $\beta$-lactams and macrolides were around $0 \%$ and $15 \%$, respectively, among penicillin-susceptible strains, around $80 \%$ for cefaclor, $50 \%$ for cefuroxime axetil, and $60 \%$ for macrolides among penicillin-intermediate strains, and around $35 \%$ for amoxicillin/clavulanic acid, 100\% for cefaclor and cefuroxime axetil, 15\% for cefotaxime, and $60 \%$ for macrolides among penicillin-resistant strains. ${ }^{27}$

Focusing on invasive isolates, the increase in the prevalence of certain serotypes and of penicillin nonsusceptibility, which was reported in the 1980s and 1990s in relation to antibiotic consumption, reversed in the 2000s when PCV7 was introduced for immunization of children. ${ }^{28,29}$ The introduction of PCV7 has not only dramatically reduced the incidence of invasive pneumococcal disease but also markedly decreased PCV7 serotypes, thus affecting the rate of penicillin and erythromycin nonsusceptibility in invasive isolates both from children and adults. ${ }^{29,30}$ The SAUCE studies in Spain (1996-1997, 1998-1999, 2001-2002, 2006-2007) have shown a decreasing trend for penicillin nonsusceptibility rates $(60.0 \%, 50.2 \%$, $43.9 \%$, and $22.9 \%$, respectively) in contrast to erythromycin nonsusceptibility, which has shown lower variations $(36.5 \%$, $34.9 \%, 34.5 \%$, and $22.9 \%$, respectively), the $\mathrm{MLS}_{\mathrm{B}}$ resistance phenotype decreasing over time (from $98.4 \%$ in 1996-1997 to $81.3 \%$ in $2006-2007$ ). Of all invasive isolates received in the Spanish Reference Laboratory for Pneumococci in 2009, $85.3 \%$ belonged to serotypes not included in PCV7, around $20 \%$ being nonsusceptible to penicillin $(\approx 50 \%$ of them serotype 19A). ${ }^{31}$ Although serotypes 1 and 19A showed significant increasing trends, only serotype 19A was linked to penicillin nonsusceptibility. ${ }^{31-33}$ In this sense, in parallel to the increasing trend for serotype $19 \mathrm{~A}^{28,32}$ there was an increasing trend for penicillin nonsusceptibility in serotype $19 \mathrm{~A}^{28}$ which reached around $80 \%$ in 2008 and was associated with amoxicillin and cefotaxime nonsusceptibility in around $20 \%$ of isolates. ${ }^{32}$

The emergence of amoxicillin resistance in pre-existing penicillin-resistant clones has been reported among PCV7 and non-PCV7 serotypes, ${ }^{34,35}$ with troublesome clones $\left(\right.$ Spain $^{23 \mathrm{~F}}-1$, Spain ${ }^{9 \mathrm{~V}}-3$, Spain ${ }^{6 \mathrm{~B}}-2$ and Spain $\left.{ }^{14}-5\right)$ exhibiting MIC values of amoxicillin higher than of penicillin. ${ }^{34}$ In addition, in 2008 a published article described in 9 Romanian isolates of the sequence type ST321 (or the nearest matched type with only a single or double locus variance), belonging to serotypes $23 \mathrm{~F}$ or $19 \mathrm{~A}$, the presence of a new cluster in regions 595-600 of PBP2X together with a new murM allele (with absence of mutations in the conserved domain of PBP1A). ${ }^{35}$ These isolates showed MICs of penicillin, amoxicillin, and cefotaxime of $\geq 16 \mu \mathrm{g} / \mathrm{mL},{ }^{35,36}$ representing a breakthrough increase in the magnitude of resistance to $\beta$-lactams. 
Also of great concern is the existence of isolates showing multiple resistance defined as full resistance to 2 or more of the 6 classes of antibacterials represented by penicillin, erythromycin, cefuroxime, tetracyclines, trimethoprim/ sulfamethoxazole, and levofloxacin. Multiple resistance in S. pneumoniae has been reported at rates as high as $19.1 \%$ in North America, 27.7\% in Western Europe, and 80.4\% in the Far East. ${ }^{25}$

According to the SAUCE study in 2006-2007, no differences in susceptibility rates were found by applying PK/PD and CLSI breakpoints, with susceptibility rates to macrolides of $\approx 80 \%$, to amoxicillin/clavulanic acid of $\approx 95 \%$, to cefuroxime axetil of $\approx 95 \%$, and to cefotaxime of $\approx 100 \% .^{15}$

\section{Cefditoren: in vitro activity, pharmacokinetics, and pharmacodynamics}

Cefditoren is the active form of cefditoren pivoxil, an oral third-generation aminothiazolyl cephalosporin with structural components similar to those of first- and third-generation cephalosporins. ${ }^{37}$ In cefditoren, the group attached at the C-7 position of the cephem skeleton affords activity against Gram-negative microorganisms, whereas the one attached at the C-3 position (not seen in other nonfirst-generation cephalosporins) affords activity against Gram-positive bacteria. ${ }^{37}$ According to the study by Yamada et al on the structure of the PBP2X of S. pneumoniae complexed with cefditoren, the unique methylthiazole group of the C-3 side chain of the cephem skeleton fits well into the PBP binding pocket, a feature that is likely to play a role in the high activity of cefditoren against $S$. pneumoniae. ${ }^{38}$

\section{In vitro activity}

Cefditoren exhibited high intrinsic activity against $S$. pyogenes, with $\mathrm{MIC}_{50} / \mathrm{MIC}_{90}$ values of $\leq 0.03 / \leq 0.03 \mu \mathrm{g} / \mathrm{mL}$, both against macrolide-susceptible or -resistant strains, in a multicenter, multinational study performed in Europe. ${ }^{14}$ The intrinsic activity of cefditoren was similar against $H$. influenzae $\left(\mathrm{MIC}_{50} /\right.$ $\mathrm{MIC}_{90}=\leq 0.03 / \leq 0.03 \mu \mathrm{g} / \mathrm{mL}$ ), as shown in a study carried out in 8 Central and Eastern European countries in 2005-2006. ${ }^{39}$ This in vitro activity against $H$. influenzae was not influenced by the phenotype/genotype of the isolates, with similar intrinsic activity to cefotaxime against ampicillin-susceptible, beta-lactamase positive, BLNAR, and BLPACR strains $\left(\mathrm{MIC}_{90}\right.$ values ranging from $\leq 0.015$ to $0.06 \mu \mathrm{g} / \mathrm{mL}$ ). ${ }^{21,40}$

For $S$. pneumoniae the multicenter, multinational ARISE study carried out in Europe in the pre-PCV7 era (thus including a large number of isolates from serotypes
$6,9,14$, and 23, traditionally linked to resistance) showed $\mathrm{MIC}_{50} / \mathrm{MIC}_{90}$ values of cefditoren of $\leq 0.03 / 0.5 \mu \mathrm{g} / \mathrm{mL} .{ }^{41}$ After PCV7 introduction the prevalence of vaccine serotypes greatly decreased (together with a decrease in penicillin nonsusceptibility), ${ }^{28,29}$ and in the most recent SAUCE study (2006-2007) $\mathrm{MIC}_{50} / \mathrm{MIC}_{90}$ values of cefditoren were $\leq 0.015 / 0.125 \mu \mathrm{g} / \mathrm{mL} .{ }^{15}$ In parallel there has been a high increase in the prevalence of non-PCV7 serotypes ${ }^{28,29}$ which occupied the niche left by PCV7 serotypes, leading to a prevalence of $85.3 \%$ of non-PCV7 serotypes isolates among invasive isolates in 2009 in Spain. ${ }^{31}$ Of these, $80 \%$ were susceptible to penicillin ( $\mathrm{MIC}_{90}$ of cefditoren $\leq 0.06 \mu \mathrm{g} / \mathrm{mL}$ ) and $20 \%$ were nonsusceptible (of these, $56.3 \%$ for serotype 19A, $9.8 \%$ for serotype $24 \mathrm{~F}, 7.0 \%$ for serotype $35 \mathrm{~B}, 5.4 \%$ for serotype $6 \mathrm{~A}$, and $4.4 \%$ for serotypes $11 \mathrm{~A}, 15 \mathrm{~A}$, and $23 \mathrm{~B}$ each). ${ }^{31}$ The intrinsic activity of cefditoren against these penicillin nonsusceptible non-PCV7 serotypes varied on serotype basis: $\mathrm{MIC}_{90}$ of $1 \mu \mathrm{g} / \mathrm{mL}$ for penicillin-resistant and $0.5 \mu \mathrm{g} / \mathrm{mL}$ for penicillin-intermediate serotype 19A isolates, $0.5 \mu \mathrm{g} / \mathrm{mL}$ for serotype $11 \mathrm{~A}, 0.25 \mu \mathrm{g} / \mathrm{mL}$ for serotypes $35 \mathrm{~B}$, $6 \mathrm{~A}$, and $15 \mathrm{~A}, 0.12 \mu \mathrm{g} / \mathrm{mL}$ for serotype $24 \mathrm{~F}$ and $0.06 \mu \mathrm{g} / \mathrm{mL}$ for serotype $23 \mathrm{~B}$ isolates. ${ }^{31}$

The intrinsic activity of cefditoren was markedly higher than that of other $\beta$-lactams against isolates with resistance patterns highly troubling as strains belonging to clones Spain $^{23 \mathrm{~F}}-1$, Spain ${ }^{9 \mathrm{~V}}-3$, Spain ${ }^{6 \mathrm{~B}}-2$, and Spain ${ }^{14}-5$. Against these strains cefditoren showed $\mathrm{MIC}_{90}$ values $\leq 1 \mu \mathrm{g} / \mathrm{mL}, 1$ dilution lower than values for cefotaxime. ${ }^{34}$ The intrinsic activity of cefditoren against the exceptional strains of sequence type ST321 (serotypes 23F or 19A) with a new cluster in regions 595-600 of PBP2X and a new murM allele warrants special attention. ${ }^{35}$ These isolates, representing the isolates most resistant to $\beta$-lactams described in the literature, were inhibited by $2 \mu \mathrm{g} / \mathrm{mL}$ of cefditoren in contrast to MICs $\geq 16 \mu \mathrm{g} / \mathrm{mL}$ of penicillin, amoxicillin, and cefotaxime. ${ }^{35}$

No inoculum effect was observed with cefditoren when testing several clinical isolates of S. pneumoniae and H. influenzae by determining its in vitro activity using inoculum sizes of $10^{4}$ to $10^{5}$ colony forming units $(\mathrm{cfu}) / \mathrm{mL}$ compared with the one obtained with inocula of $10^{7}$ to $10^{8} \mathrm{cfu} / \mathrm{mL}{ }^{42}$

\section{Pharmacokinetics}

When orally administered to humans the prodrug cefditoren pivoxil is completely and rapidly hydrolyzed to cefditoren, the bioavailability being low in the fasting state (15\%-20\%). However when administered after high fat meals the bioavailability of cefditoren increases, $\mathrm{C}_{\max }$ and AUC values being $50 \%$ and $70 \%$, respectively, higher than those determined 
in the fasting state. ${ }^{37}$ In a phase I study in Caucasians administed $400 \mathrm{mg}$ cefditoren pivoxil single dose in the fed state, $\mathrm{C}_{\max }$ was $3.7 \pm 0.7 \mu \mathrm{g} / \mathrm{mL}, \mathrm{T}_{\max }$ was 2 hours, $\mathrm{AUC}_{\infty}$ was $12.5 \pm 1.6 \mu \mathrm{g} \times \mathrm{h} / \mathrm{mL}$, and $\mathrm{t}_{1 / 2}$ was $1.54 \pm 0.20$ hours. $^{43}$

\section{Pharmacodynamics}

By relating pharmacokinetic variables and susceptibility data (ie, antibiotic drug exposure relative to in vitro MIC), PK/ PD breakpoints indicate the highest MIC that produces the adequate value for the relevant $\mathrm{PK} / \mathrm{PD}$ parameter predicting bacterial killing, eradication, and clinical outcome. In the case of cefditoren, pharmacodynamics in relation to communityacquired respiratory tract infections should be studied especially for $S$. pneumoniae, since $H$. influenzae and $S$. pyogenes are uniformly and highly susceptible to cefditoren. For cefditoren, as for $\beta$-lactams, the time ( $\mathrm{t}$ ) (expressed as percentage of the dosing interval) that antibiotic concentrations exceed the value of MIC $(\mathrm{t}>\mathrm{MIC})$ is the index predicting efficacy with a cut-off value of $40 \%$ for clinical cure in humans, ${ }^{44,45}$ although a $\mathrm{t}>\mathrm{MIC}$ of $33 \%$ has been used as bacteriostatic endpoint and susceptibility is defined by the FDA and CLSI as inhibition by usually achievable concentrations of the recommended dose. ${ }^{17,46}$

Data from a phase I study in Caucasians showed that the $400 \mathrm{mg}$ twice daily regimen of cefditoren pivoxil gave a value of $\mathrm{t}>$ MIC for total drug of about $55 \%$ for MIC of $0.5 \mu \mathrm{g} / \mathrm{mL}, 68 \%$ for MIC of $0.25 \mu \mathrm{g} / \mathrm{mL}, 81 \%$ for MIC of $0.12 \mu \mathrm{g} / \mathrm{mL}$, and $94 \%$ for MIC of $0.06 \mu \mathrm{g} / \mathrm{mL} .{ }^{43}$ Classically the unbound fraction of an antimicrobial has been considered the active fraction in vitro; however the reversibility of protein binding implies that limitation of activity may be far from absolute, even in highly protein bound agents. ${ }^{47}$ For cefditoren, which exhibits $88 \%$ protein binding, ${ }^{37}$ this could be postulated as a limitation to its in vivo intrinsic activity. Considering that protein binding rates in humans and mice are equal for cefditoren, the extrapolation to humans of pharmacodynamic data obtained in animal models acquires special interest. A pneumococcal sepsis model in mice infected by strains with exceptionally high cefditoren MIC $(1-2 \mu \mathrm{g} / \mathrm{mL}$ ) showed that cefditoren $\mathrm{t}>\mathrm{MIC}$ of $\approx 35 \%$ (free $\mathrm{t}>$ MIC of $\approx 20 \%$ ) produced $100 \%$ survival in contrast to placebo $\left(0 \%\right.$ survival).$^{48}$ Free $\mathrm{t}>\mathrm{MIC}$ of $\approx 20 \%$ was also related to $>99.9 \%$ reduction in bacterial load (of 2 isolates serotypes $6 \mathrm{~B}$ and $15 \mathrm{~A}$ with $\mathrm{MIC}=0.25 \mu \mathrm{g} / \mathrm{mL}$ ) in an in vitro pharmacodynamic simulation with physiological albumin concentrations and $86 \%$ protein binding rate in the device. ${ }^{49}$ In a cefditoren Monte Carlo simulation (using data determined in the phase I study ${ }^{43}$ ) considering 33\% $\mathrm{t}>\mathrm{MIC}$ (bacteriostatic endpoint) as endpoint value (approximately the value obtained in the mice model for total drug), strains with MICs up to $0.5 \mu \mathrm{g} / \mathrm{mL}$ were covered by cefditoren. ${ }^{46}$ When applying free drug 33\% $\mathrm{t}>\mathrm{MIC}$ (a value higher than the one required in the mice model for $100 \%$ survival and in the pharmacodynamic simulation for $>99.9 \%$ reduction in bacterial load), strains up to $0.25 \mu \mathrm{g} / \mathrm{mL}$ were covered. ${ }^{46}$ If the classical theoretical value of free $\mathrm{t}>\mathrm{MIC}$ of $40 \%$ was considered, strains up to $0.12 \mu \mathrm{g} / \mathrm{mL}$ were covered by cefditoren in the Monte Carlo simulation. ${ }^{46}$

According to these data, the cefditoren breakpoint for nonsusceptibility should be within the range $\leq 0.12 \mu \mathrm{g} / \mathrm{mL}$ and $\leq 0.5 \mu \mathrm{g} / \mathrm{mL}$. This latter value has been proposed by several authors considering cefditoren $\mathrm{MIC}_{90}$ values lower than the breakpoints values for parenteral thirdgeneration cephalosporins and the pharmacokinetics of cefditoren, ${ }^{50-52}$ and was the breakpoint approved by the Spanish Agency during the registration procedure in Europe (susceptibility $\leq 0.5 \mu \mathrm{g} / \mathrm{mL}$ ). ${ }^{53}$ The most conservative breakpoint $\left(\leq 0.12 \mu \mathrm{g} / \mathrm{mL}\right.$ ) has been proposed by the FDA. ${ }^{54}$ With this breakpoint, $94.9 \%$ of $S$. pneumoniae isolates in Spain in 2006-2007 are covered. ${ }^{15}$

An interesting pharmacodynamic result for cefditoren in the above-mentioned mice model was the decrease in the required $\mathrm{t}>\mathrm{MIC}$ values producing efficacy when animals were passively immunized (with nonprotective levels of specific antibodies) prior to infection. The required values of $\mathrm{t}>$ MIC linked to efficacy decreased from $\approx 35 \%$ (free $\mathrm{t}>\mathrm{MIC}$ of $\approx 20 \%$ ) in nonimmunized animals to $\approx 25 \%$ (free $\mathrm{t}>\mathrm{MIC}$ of $\approx 15 \%$ ) in the immunized ones. ${ }^{48}$ This is interesting in the context where specific antibodies (at nonprotective levels) are likely to be present before $S$. pneumoniae infection, since colonization is to some extent a B-cell immunizing event ${ }^{55,56}$ and preventive measures such as pneumococcal vaccination are increasingly been used in the community. The benefit of these findings would not be the decrease of administered doses, but rather the coverage by current regimens of the exceptional strains with high MIC values.

Cefditoren has shown a postantibiotic effect greater than 1 hour for $S$. pneumoniae and $S$. pyogenes, which also supports the twice daily regimen for the treatment of respiratory tract infections..$^{57}$

\section{Efficacy clinical studies with cefditoren in the treatment of upper respiratory tract infections}

During the clinical development of cefditoren, 3 comparative multicenter studies were performed with cefditoren 
and penicillin $\mathrm{V}$ as comparator in pharyngotonsillitis in patients $\geq 12$ years of age. The three studies included a total of 1322 randomized patients, with 1241 patients in the intention-to-treat (ITT) analysis and 1010 patients in the perprotocol (PP) population. In the pooled analysis of the three studies, no significant differences in clinical response were found between cefditoren and penicillin $\mathrm{V}$, success rates ranging from $89.4 \%$ to $95.3 \%{ }^{58}$ Eradication of $S$. pyogenes was higher with cefditoren at the end of therapy $(90.4 \%$ vs $82.7 \% ; P=0.002)$ and at the end of follow-up $(84.7 \%$ vs $76.7 \% ; P=0.008)$, although the statistical significance set at $P<0.001$ was not reached. ${ }^{58}$

Two additional studies have been recently published for cefditoren in the treatment of group A $\beta$-hemolytic streptococcal pharyngotonsillitis in children. ${ }^{59,60}$ Ozaki et al reported a study comparing $3 \mathrm{mg} / \mathrm{kg}$ cefditoren-pivoxil 3 times daily for 5 days versus amoxicillin $10 \mathrm{mg} / \mathrm{kg} 3$ times daily for 10 days in 258 patients (258 isolates with amoxicillin and cefditoren $\mathrm{MIC} \leq 0.06 \mu \mathrm{g} / \mathrm{mL}) .{ }^{59}$ Eradication was $100 \%$ with amoxicillin and 99\% with cefditoren pivoxil, with no differences in the recurrence rate (15 patients in the amoxicillin arm and 8 patients in the cefditoren arm). ${ }^{59}$ The second study compared the efficacy provided by $9 \mathrm{mg} / \mathrm{kg}$ cefditoren pivoxil (maximum dose $300 \mathrm{mg} /$ day) 3 times daily for 5 days (77 patients) versus 10 days (149 patients) in children with history of at least 1 episode of group A $\beta$-hemolytic streptococcal pharyngotonsillitis. ${ }^{60}$ Similar bacteriological failure occurred in both arms (9.1\% vs $11.4 \%)$, but significantly higher recurrences were found in the 5 days treatment group $(9.1 \%$ vs $0.7 \% ; P=0.03) .{ }^{60}$

Three studies were carried out in adults with acute sinusitis during the clinical development of cefditoren, using cefuroxime or amoxicillin/clavulanic acid as comparators. The three studies included 1819 randomized patients: 1726 patients included in the ITT and 1589 patients in the PP population. In the pooled analysis of the three studies, no differences in clinical response were found between cefditoren and comparators both at the end of therapy (80.2\% vs $84.8 \%)$ and at the end of follow-up $(71.2 \%$ vs $77.4 \%) .{ }^{58}$ An additional study in the treatment of bacterial rhinosinusitis in children was recently published comparing 4 to $6 \mathrm{mg} / \mathrm{kg}$ twice daily (maximum dose $300 \mathrm{mg} /$ day) versus 80 to $90 \mathrm{mg} / \mathrm{kg}$ (on amoxicillin basis) amoxicillin/clavulanic acid (maximum dose $800 \mathrm{mg} /$ day) twice daily for 14 days. ${ }^{61}$ Rates of improvement were similar in both groups $(78.8 \%$ vs $84.7 \%)$ with similar relapse rates $(9.1 \%$ vs $11.1 \%)$ or recurrences $(3.0 \%$ vs $5.6 \%)$ of sinus symptoms. ${ }^{61}$

\section{Efficacy clinical studies with cefditoren in the treatment of community-acquired lower respiratory tract infections}

Seven studies were carried out in the clinical development of cefditoren in the treatment of lower respiratory tract infections, 4 studies in community-acquired pneumonia (CAP) and 3 studies in acute exacerbation of chronic bronchitis (AECB). A pooled analysis of data was performed including a total of 4159 randomized patients. ${ }^{62}$ Comparators were amoxicillin/clavulanic acid or cefpodoxime in CAP studies, and cefuroxime or clarithromycin in AECB studies. Pooled data from CAP studies revealed no significant differences in response rates between cefditoren and comparators, percentages of responders ranging from $89.2 \%$ to $91.8 \%$ at the end of therapy and from $85.9 \%$ to $90.4 \%$ at the end of follow-up. ${ }^{62}$ Pooled data from the AECB studies resulted in response rates ranging from $85.8 \%$ to $91.3 \%$ at the end of therapy and from $81.2 \%$ to $83.3 \%$ at the end of follow-up, without significant differences between groups. ${ }^{6}$

For microbiological assessment, data from CAP and AECB studies were pooled. Globally, the bacteriologically evaluable population consisted of 1910 patients with 1223 target pathogens isolated prior to treatment initiation, including $406 S$. pneumoniae isolates (56 of them nonsusceptible to oral penicillin) and $595 \mathrm{H}$. influenzae isolates. ${ }^{62}$ No significant differences were found in the rate of bacteriological responders for S. pneumoniae, percentages ranging from $88.5 \%$ to $92.0 \%$. Among penicillin nonsusceptible (MIC $\geq 0.12 \mu \mathrm{g} / \mathrm{mL})$ S. pneumoniae isolates, all $20(100 \%)$ strains in the cefditoren $400 \mathrm{mg}$ group, $84.2 \%$ (16 out of 19) strains in the cefditoren $200 \mathrm{mg}$ group, and $94.1 \%$ (16 out of 17) strains in the comparator group were eradicated or presumed eradicated. ${ }^{62}$ Among penicillin-resistant (MIC $\geq 2 \mu \mathrm{g} / \mathrm{mL}$ ) isolates, 17 (94.4\%) of 18 isolates in both cefditoren arms were eradicated or presumed eradicated, compared with 10 (90.9\%) of 11 in the comparator group. ${ }^{62}$ Differences in the rate of responders for H. influenzae were also not significant (range $82.7 \%$ to $86.6 \%$ ). ${ }^{62}$

\section{Bacterial eradication as a goal of antibacterial treatment, and subsequent clinical efficacy}

The correlation between bacterial eradication and clinical outcome in respiratory tract infections can be explored in streptococcal tonsillopharyngitis as an upper respiratory tract infection and AECB as a lower respiratory tract infection, 
since noninvasive samples for culture are easily collected over time. As noted in the previous section, in the pooled analysis of studies on tonsillopharyngitis bacteriological efficacy was associated with a higher clinical response rate. ${ }^{58}$ Cefditoren (a $\beta$-lactamase-resistant cephalosporin) showed a tendency of higher bacteriological response for $S$. pyogenes than penicillin (susceptible to $\beta$-lactamases produced by commensal flora), ${ }^{58}$ reinforcing the concept of "co-pathogenicity" with $\beta$-lactamase-producing bacteria. It has been hypothesized that $\beta$-lactamase from $\beta$-lactamaseproducing isolates such as $H$. influenzae may protect non$\beta$-lactamase-producing isolates such as $S$. pyogenes from the action of the antibiotic. ${ }^{63,64}$ In this sense, a previous meta-analysis also showed higher bacteriological failures with penicillins versus cephalosporins ${ }^{65}$ Co-pathogenicity was also explored in vitro in a pharmacodynamic simulation of free concentrations over 24 hours obtained with $875 \mathrm{mg}$ amoxicillin 3 times daily, 875/125 mg amoxicillin/clavulanic acid 3 times daily, and $400 \mathrm{mg}$ cefditoren twice daily using individual and mixed inocula (proportions 1:1:1:1) of 1 S. pyogenes isolate, 1 penicillin-resistant S. pneumoniae, $1 \beta$-lactamase positive $H$. influenzae, and 1 BLPACR H. influenzae isolate. ${ }^{66}$ At 24 hours amoxicillin was able to eradicate $S$. pyogenes in simulations carried out with the individual $S$. pyogenes inoculum but failed to do so in simulations with the mixed inocula where $S$. pyogenes was protected by $\beta$-lactamases produced by the $H$. influenzae isolates. ${ }^{66}$ In contrast $S$. pyogenes was eradicated by cefditoren, both in individual and mixed simulations where both strains of $H$. influenzae were also eradicated. ${ }^{66}$

One of the AECB studies included in the pooled analysis of lower respiratory tract infections ${ }^{62}$ exploring the relationship of bacteriological and clinical responses has been individually published. ${ }^{67}$ In this study, including 541 patients, inclusion criteria, baseline characteristics of patients (age and pre-study smoking history), and the required symptoms of exacerbation (Anthonisen I and II) ensured inclusion of patients with underlying chronic bronchitis, and increased the chance of the bacterial origin of the exacerbation. Improvement/efficacy was more evident in signs linked to the presence of bacteria, such as rales, ronchi, and the volume and purulence of the sputum (which decreased from about $80 \%$ patients in the pre-treatment visit to about $10 \%$ patients at day 30) than in signs linked to chronic structural damage such as dyspnea which decreased from only about $99 \%$ in the pre-treatment visit to $75 \%$ patients at day $30 .{ }^{67}$ Globally, perpathogen bacterial response related well to clinical success: $83.5 \%$ isolates (out of 164 baseline isolates) from patients resulting in clinical response were eradicated or presumably eradicated in contrast to only $3 \%$ isolates (out of 33 baseline isolates) from patients resulting in clinical failure. ${ }^{67}$

\section{Safety data from clinical trials performed during the clinical development of cefditoren}

Safety data from all the 13 clinical trials carried out during the clinical development of cefditoren for the treatment of community-acquired respiratory infections were analyzed in a pooled analysis ${ }^{68}$ Safety population was defined as all randomized patients with at least 1 dose intake, and comprised 4592 patients for cefditoren. ${ }^{68}$ Cefditoren exhibited an adverse event profile similar to that of previous antibiotics currently used in the treatment of community-acquired respiratory tract infections, diarrhea being the most frequent adverse event $(9.9 \%)$ followed by vaginosis $(3.9 \%$ among female population), nausea (3.5\%), abdominal pain (1.8\%), and dyspepsia $(1.1 \%){ }^{68}$

\section{Conclusions}

The pharmaceutical industry has decreased its efforts to develop new antimicrobials, and the lack of a robust pipeline of new compounds has been suggested. ${ }^{69}$ In this situation, the fact that the main ecological effect of antimicrobials is promoting the emergence of resistance ${ }^{70}$ (a risk associated with the imprudent use of available drugs) implies that clinicians may have limited options within the current armamentarium. Since the use of agents to which pathogens are resistant contributes to increased morbidity, mortality, and associated expenses, ${ }^{71}$ the rational use of antibiotics can help to stop or minimize the emergence, spread, and diversification of antibiotic resistance.

In this context, this article reviews and updates published data on cefditoren in the evolving situation of resistance among the most prevalent isolates from respiratory tract infections in the community. By relating its in vitro activity (from surveillance and in vitro studies including isolates with emerging resistant genotypes/phenotypes) to its pharmacokinetics, the cefditoren pharmacodynamic activity predicting therapeutic efficacy (in humans, animal models, and in vitro simulations) was analyzed prior to reviewing clinical studies and the relationship between bacterial eradication and therapeutic efficacy. The wide range of studies carried out with cefditoren constitutes one of the most extensive and continuous sources of data defining the role of a new antibiotic in the community and provides information to clinicians (on cefditoren and comparators) of relevance to establish empirical therapy. 
The high in vitro activity of cefditoren against the most prevalent respiratory isolates in the community, together with its pharmacokinetics (allowing a twice daily regimen) leading to adequate pharmacodynamic indexes covering all $S$. pyogenes, $H$. influenzae, and 94.9\% S. pneumoniae isolates, makes cefditoren an antibiotic that will play a significant role in the treatment of respiratory tract infections in the community. In the clinical setting, studies carried out with cefditoren showed that treatments with the $400 \mathrm{mg}$ twice daily regimen were associated with high rates of bacteriological response, even against penicillin nonsusceptible $S$. pneumoniae, with good correlation between bacteriological efficacy/response and clinical outcome.

\section{Disclosure}

The authors declare no conflicts of interest.

\section{References}

1. Mogyoros M. Challenges of managed care organisations in treating respiratory tract infections in an age of antibiotic resistance. Am J Man Care. 2001;7(Suppl 6):163-169.

2. Llor C. Consideraciones a la hora de la prescripción antibiótica en atención primaria. Med Clin Monogr (Barc). 2004;5:52-57.

3. Huovinen P, Cars O. Control of antimicrobial resistance: time for action. The essentials of control are already well known. BMJ. 1998; 317:613-614.

4. García-Rey C, Fenoll A, Aguilar L, Casal J. Effect of social and climatological factors on antimicrobial use and Streptococcus pneumoniae resistance in different provinces in Spain. J Antimicrob Chemother. 2004;54:465-471

5. Granizo JJ, Aguilar L, Casal J, García-Rey C, Dal-Ré R, Baquero F. Streptococcus pneumoniae resistance to erythromycin and penicillin in relation to macrolide and beta-lactam consumption in Spain (1979-1997). J Antimicrob Chemother. 2000;46:767-773.

6. García-Rey C, Aguilar L, Baquero F, Casal J, Dal-Ré R. Importance of local variations in antibiotic consumption and geographical differences of erythromycin and penicillin resistance in Streptococcus pneumoniae. J Clin Microbiol. 2002;40:159-164.

7. Gómez J, Ruiz-Gómez J, Hernández-Cardona JL, Nuñez ML, Canteras M, Valdés M. Antibiotic resistance patterns of Streptococcus pneumoniae, Haemophilus influenzae and Moraxella catarrhalis: a prospective study in Murcia, Spain, 1983-1992. Chemotherapy. 1994;40:299-303.

8. Granizo JJ, Aguilar L, Casal J, Dal-Ré R, Baquero F. Streptococcus pyogenes resistance to erythromycin in relation to macrolide consumption in Spain (1986-1997). J Antimicrob Chemother. 2000;46: 959-964.

9. García-Rey C, Aguilar L, Baquero F, Casal J, Martín JE. Pharmacoepidemiological analysis of provincial differences between consumption of macrolides and rates of erythromycin resistance among Streptococcus pyogenes isolates in Spain. J Clin Microbiol. 2002;40:2959-2963.

10. Pérez-Trallero E, García-de-la-Fuente C, García-Rey C, et al. Geographical and ecological analysis of resistance, coresistance, and coupled resistance to antimicrobials in respiratory pathogenic bacteria in Spain. Antimicrob Agents Chemother. 2005;49:1965-1972.

11. Gómez-Lus R, Granizo JJ, Aguilar L, Bouza E, Gutierrez A, García-de-Lomas J. Is there an ecological relationship between rates of antibiotic resistance of species of the genus Streptococcus? The Spanish Surveillance Group for Respiratory Pathogens. J Clin Microbiol. 1999;37:3384-3386.
12. Richter SS, Heilmann KP, Beekmann SE, et al. Macrolide-resistant Streptococcus pyogenes in the United States, 2002-2003. Clin Infect Dis. 2005;41:599-608.

13. Chan JC, Chu YW, Chu MY, Cheung TK, Lo JY. Epidemiological analysis of Streptococcus pyogenes infections in Hong Kong. Pathology. 2009;41:681-686.

14. Gracia M, Díaz C, Coronel P, et al. Antimicrobial susceptibility of Streptococcus pyogenes in Central, Eastern, and Baltic European Countries, 2005 to 2006: the cefditoren surveillance program. Diagn Microbiol Infect Dis. 2009;64:52-56.

15. Pérez-Trallero E, Martín-Herrero JE, Mazón A, et al. Antimicrobial resistance among respiratory pathogens in Spain: latest data and changes over 11 years (1996-1997 to 2006-2007). Antimicrob Agents Chemother. 2010;54:2953-2959.

16. Prieto J, Calvo A, Gómez-Lus ML. Antimicrobial resistance: a class effect? J Antimicrob Chemother. 2002;50(Suppl S2):7-12.

17. Clinical and Laboratory Standards Institute. Performance standards for antimicrobial susceptibility testing; nineteenth informational supplement. CLSI document M100-S19. Clinical and Laboratory Standards Institute, Wayne, PA, USA; 2009.

18. Sahm DF, Brown NP, Thornsberry C, Jones ME. Antimicrobial susceptibility profiles among common respiratory tract pathogens: a GLOBAL perspective. Postgrad Med. 2008;120(3 Suppl 1):16-24.

19. Sahm DF, Brown NP, Draghi DC, Evangelista AT, Yee YC, Thornsberry $\mathrm{C}$. Tracking resistance among bacterial respiratory tract pathogens: summary of findings of the TRUST Surveillance Initiative, 2001-2005. Postgrad Med. 2008;120(3 Suppl 1):8-15.

20. Jansen WT, Verel A, Beitsma M, Verhoef J, Milatovic D. Longitudinal European surveillance study of antibiotic resistance of Haemophilus influenzae. J Antimicrob Chemother. 2006;58:873-877.

21. Sevillano D, Giménez MJ, Cercenado E, et al. Genotypic versus phenotypic characterization, with respect to beta-lactam susceptibility, of Haemophilus influenzae isolates exhibiting decreased susceptibility to beta-lactam resistance markers. Antimicrob Agents Chemother. 2009;53:267-270.

22. Niki Y, Hanaki H, Matsumoto $T$, et al. Nationwide surveillance of bacterial respiratory pathogens conducted by the Japanese Society of Chemotherapy in 2007: general view of the pathogens' antibacterial susceptibility. J Infect Chemother. 2009;15:156-167.

23. Sakata H, Toyonaga Y, Sato Y, et al. Nationwide survey of the development of drug-resistance in the pediatric field: drug sensitivity of Haemophilus influenzae in Japan. J Infect Chemother. 2009;15: 402-409.

24. Peric M, Bozdogan B, Jacobs MR, Appelbaum PC. Effects of an efflux mechanism and ribosomal mutations on macrolide susceptibility of Haemophilus influenzae clinical isolates. Antimicrob Agents Chemother. 2003;47:1017-1022.

25. Cantón R, Unal S, Farrell DJ. Antibacterial resistance patterns in Streptococcus pneumoniae isolated from elderly patients: PROTEKT years 1-5 (1999-2004). Int J Antimicrob Agents. 2007;30:546-550.

26. Darabi A, Hocquet D, Dowzicky MJ. Antimicrobial activity against Streptococcus pneumoniae and Haemophilus influenzae collected globally between 2004 and 2008 as part of the Tigecycline Evaluation and Surveillance Trial. Diagn Microbiol Infect Dis. 2010;67: $78-86$.

27. Pérez-Trallero E, García-de-la-Fuente C, García-Rey C, et al. Geographical and ecological analysis of resistance, coresistance, and coupled resistance to antimicrobials in respiratory pathogenic bacteria in Spain. Antimicrob Agents Chemother. 2005;49:1965-1972.

28. Fenoll A, Granizo JJ, Aguilar L, et al. Temporal trends of invasive Streptococcus pneumoniae serotypes and antimicrobial resistance patterns in Spain from 1979 to 2007. J Clin Microbiol. 2009;47:1012-1020.

29. Fenoll A, Aguilar L, Granizo JJ, et al. Has the licensing of respiratory quinolones for adults and the 7-valent pneumococcal conjugate vaccine (PCV-7) for children had herd effects with respect to antimicrobial non-susceptibility in invasive Streptococcus pneumoniae? J Antimicrob Chemother. 2008;62:1430-1433. 
30. Whitney CG, Farley MM, Hadler J, et al. Decline in invasive pneumococcal disease after the introduction of protein-polysaccharide conjugate vaccine. $N$ Engl J Med. 2003;348:1737-1746.

31. Fenoll A, Aguilar L, Giménez MJ, et al. Susceptibility of recently collected Spanish pneumococci nonsusceptible to oral penicillin from serotypes not included in the 7-valent conjugate vaccine. Antimicrob Agents Chemother. 2010;54:2696-2698.

32. Fenoll A, Aguilar L, Vicioso MD, et al. Serotype distribution and susceptibility of Streptococcus pneumoniae isolates from pleural fluid in the current decade in Spain. Antimicrob Agents Chemother. 2010;54:5387-5390.

33. Picazo J, Ruiz-Contreras J, Casado-Flores J, et al. Relations between serotypes, age and clinical presentation of invasive pneumococcal disease in Madrid after the introduction of the 7-valent pneumococca conjugate vaccine into the vaccination calendar. Clin Vaccine Immunol. 2011;18:89-94.

34. Pérez-Trallero E, Marimón JM, Ercibengoa M, Giménez MJ, Coronel P, Aguilar L. Antimicrobial susceptibilities of amoxycillin-non-susceptible and susceptible isolates among penicillin-non-susceptible Streptococcus pneumoniae. Clin Microbiol Infect. 2007;13:937-940.

35. Soriano F, Cafini F, Aguilar L, et al. Breakthrough in penicillin resistance? Streptococcus pneumoniae isolates with penicillin/cefotaxime MICs of $16 \mathrm{mg} / \mathrm{L}$ and their genotypic and geographical relatedness. J Antimicrob Chemother. 2008;62:1234-1240.

36. Rodríguez-Cerrato V, Gracia M, Del Prado G, et al. Antimicrobial susceptibility of multidrug-resistant Streptococcus pneumoniae strains with penicillin MICs of 8 to $32 \mathrm{mg} / \mathrm{L}$. Diagn Microbiol Infect Dis. 2010;66:336-338.

37. Wellington K, Curran MP. Cefditoren pivoxil: a review of its use in the treatment of bacterial infections. Drugs. 2004;64:2597-2618.

38. Yamada M, Watanabe T, Miyara T, et al. Crystal structure of cefditoren complexed with Streptococcus pneumoniae penicillin-binding protein 2X: structural basis for its high antimicrobial activity. Antimicrob Agents Chemother. 2007;51:45-50.

39. Gracia M, Díaz C, Coronel P, et al. Antimicrobial susceptibility of Haemophilus influenzae and Moraxella catarrhalis isolates in eight Central, East and Baltic European countries in 2005-06: results of the Cefditoren Surveillance Study. J Antimicrob Chemother. 2008;61:1180-1181.

40. García-de-Lomas J, Lerma M, Cebrián L, et al. Influence of Haemophilus influenzae beta-lactamase production and/or ftsI gene mutations on in vitro activity of and susceptibility rates to aminopenicillins and second- and third-generation cephalosporins. Int J Antimicrob Agents. 2007:30:190-192.

41. Soriano F, Granizo JJ, Fenoll A, et al. Antimicrobial resistance among clinical isolates of Streptococcus pneumoniae isolated in four southern European countries (ARISE project) from adult patients: results from the cefditoren surveillance program. J Chemother. 2003;15: $107-112$.

42. Soriano F, Coronel P, Gimeno M, Jiménez M, García-Corbeira P, Fernández-Roblas R. Inoculum effect and bactericidal activity of cefditoren and other antibiotics against Streptococcus pneumoniae, Haemophilus influenzae, and Neisseria meningitidis. Eur J Clin Microbiol Infect Dis. 1996;15:761-763.

43. Sádaba B, Azanza JR, Quetglas EG, et al. Pharmacokinetic/pharmacodynamic serum and urine profile of cefditoren following single-dose and multiple twice- and thrice-daily regimens in healthy volunteers: a phase I study. Rev Esp Quimioter. 2007;20:51-60.

44. Craig WA. Pharmacokinetic/pharmacodynamic parameters: rationale for antibacterial dosing of mice and men. Clin Infect Dis. 1998;26: $1-10$.

45. Heffelfinger JD, Dowell SF, Jorgensen JH, et al. Management of community-acquired pneumonia in the era of pneumococcal resistance: a report from the Drug-Resistant Streptococcus pneumoniae Therapeutic Working Group. Arch Intern Med. 2000;160:1399-1408.

46. Granizo JJ, Sádaba B, Honorato J, et al. Monte Carlo simulation describing the pharmacodynamic profile of cefditoren in plasma from healthy volunteers. Int J Antimicrob Agents. 2008;31:396-398.
47. Moellering RC, Eliopoulos GM. Principles of anti-infective therapy. In: Mandell GL, Bennett JE, Dolin R, editors. Mandell, Douglas, and Bennett Principles and Practice of Infectious Diseases, 6th ed. Philadelphia: Elsevier Churchill Livingstone; 2005:242-253.

48. Cafini F, Yuste J, Giménez MJ, et al. Enhanced in vivo activity of cefditoren in pre-immunized mice against penicillin-resistant S. pneumoniae (serotypes $6 \mathrm{~B}, 19 \mathrm{~F}$ and $23 \mathrm{~F}$ ) in a sepsis model. PLoS One. 2010;5(8):e12041.

49. Sevillano D, Aguilar L, Alou L, et al. High protein binding and cidal activity against penicillin-resistant $S$. pneumoniae: a cefditoren in vitro pharmacodynamic simulation. PLoS One. 2008;3(7):e2717.

50. Karlowsky JA, Jones ME, Draghi DC, Critchley IA, Thornsberry C, Sahm DF. In vitro susceptibility of recent clinical isolates of pneumococci to the investigational cephalosporin cefditoren. Diagn Microbiol Infect Dis. 2002;42:59-64.

51. Johnson DM, Biedenbach DJ, Beach ML, Pfaller MA, Jones RN. Antimicrobial activity and in vitro susceptibility test development for cefditoren against Haemophilus influenzae, Moraxella catarrhalis, and Streptococcus species. Diagn Microbiol Infect Dis. 2000;37:99-105.

52. Jones RN, Biedenbach DJ, Croco MA, Barrett MS. In vitro evaluation of a novel orally administered cephalosporin (Cefditoren) tested against 1249 recent clinical isolates of Haemophilus influenzae, Moraxella catarrhalis, and Streptococcus pneumoniae. Diagn Microbiol Infect Dis. 1998;31:573-578.

53. Agencia Española de Medicamentos y Productos Sanitarios. https:// sinaem4.agemed.es/consaem/fichasTecnicas.do?metodo=detalleForm.

54. Food and Drug Administration. Spectracef prescribing information. http://www.crtx.com/docs/spectracef.pdf.

55. Weinberger DM, Dagan R, Givon-Lavi N, et al. Epidemiologic evidence for serotype-specific acquired immunity to pneumococcal carriage. J Infect Dis. 2008;197:1511-1518.

56. Soininen A, Pursiainen H, Kilpi T, Käyhty H. Natural development of antibodies to pneumococcal capsular polysaccharides depends on the serotype: association with pneumococcal carriage and acute otitis media in young children. $J$ Infect Dis. 2001;184:569-576.

57. Felmingham D, Robbins MJ, Ghosh G, et al. An in vitro characterization of cefditoren, a new oral cephalosporin. Drugs Exp Clin Res. 1994;20: $127-147$.

58. Granizo JJ, Giménez MJ, Barberán J, Coronel P, Gimeno M, Aguilar L. Efficacy of cefditoren in the treatment of upper respiratory tract infections: a pooled analysis of six clinical trials. Rev Esp Quimioter. 2008;21:14-21.

59. Ozaki T, Nishimura N, Suzuki M, et al. Five-day oral cefditoren pivoxil versus 10-day oral amoxicillin for pediatric group A streptococcal pharyngotonsillitis. J Infect Chemother. 2008;14:213-218.

60. Kikuta H, Shibata M, Nakata S, et al. Comparative study of 5-day and 10-day cefditoren pivoxil treatments for recurrent group A beta-hemolytic Streptococcus pharyngitis in children. Int $J$ Pediatr. 2009:863608.

61. Poachanukoon O, Kitcharoensakkul M. Efficacy of cefditoren pivoxil and amoxicillin/clavulanate in the treatment of pediatric patients with acute bacterial rhinosinusitis in Thailand: a randomized, investigatorblinded, controlled trial. Clin Ther. 2008;30:1870-1879.

62. Granizo JJ, Giménez MJ, Barberán J, Coronel P, Gimeno M, Aguilar L. The efficacy of cefditoren pivoxil in the treatment of lower respiratory tract infections, with a focus on the per-pathogen bacteriologic response in infections caused by Streptococcus pneumoniae and Haemophilus influenzae: a pooled analysis of seven clinical trials. Clin Ther. 2006; 28:2061-2069.

63. Casey JR, Pichichero ME. The evidence base for cephalosporin superiority over penicillin in streptococcal pharyngitis. Diagn Microbiol Infect Dis. 2007;57(3 Suppl):S39-S45.

64. Budhani RK, Struthers JK. Interaction of Streptococcus pneumoniae and Moraxella catarrhalis: investigation of the indirect pathogenic role of beta-lactamase-producing moraxellae by use of a continuousculture biofilm system. Antimicrob Agents Chemother. 1998;42: 2521-2526. 
65. Casey JR, Pichichero ME. Meta-analysis of cephalosporins versus penicillin for treatment of group A streptococcal tonsillopharyngitis in adults. Clin Infect Dis. 2004;38:1526-1534.

66. Sevillano D, Aguilar L, Alou L, et al. Beta-lactam effects on mixed cultures of common respiratory isolates as an approach to treatment effects on nasopharyngeal bacterial population dynamics. PLoS One. 2008;3(12):e3846

67. Alvarez-Sala JL, Kardos P, Martínez-Beltrán J, Coronel P, Aguilar L. Clinical and bacteriological efficacy in treatment of acute exacerbations of chronic bronchitis with cefditoren-pivoxil versus cefuroxime-axetil. Antimicrob Agents Chemother. 2006;50:1762-1767.

68. Granizo JJ, Aguilar L, Gimenez MJ, Coronel P, Gimeno M, Prieto J. Safety profile of cefditoren. A pooled analysis of data from clinical trials in community-acquired respiratory tract infections. Rev Esp Quimioter. 2009;22:57-61.
69. Almirante B, Campos J, Cantón R, et al. Prudent use of antimicrobials: have we done the best we can? The SEIMC and REIPI statement. Enferm Infecc Microbiol Clin. 2010;28:485-486.

70. Baquero F, Garau J. Prudent use of antimicrobial agents: revisiting concepts and estimating perspectives in a global world. Enferm Infecc Microbiol Clin. 2010;28:487-488.

71. Daneman N, Low DE, McGeer A, Green KA, Fisman DN. At the threshold: defining clinically meaningful resistance thresholds for antibiotic choice in community-acquired pneumonia. Clin Infect Dis. 2008;46:1131-1138.

\section{Publish your work in this journal}

Drug Design, Development and Therapy is an international, peerreviewed open-access journal that spans the spectrum of drug design and development through to clinical applications. Clinical outcomes, patient safety, and programs for the development and effective, safe, and sustained use of medicines are a feature of the journal, which has also been accepted for indexing on PubMed Central. The manuscript management system is completely online and includes a very quick and fair peer-review system, which is all easy to use. Visit http://www.dovepress.com/testimonials.php to read real quotes from published authors.

Submit your manuscript here: http://www.dovepress.com/drug-design-development-and-therapy-journal 